\title{
Impact of phonon-assisted tunneling on electronic conductivity in graphene nanoribbons and oxides ones
}

\author{
Povilas Pipinys, Antanas Kiveris*
}

Department of Physics, Vilnius Pedagogical University, Vilnius, Lithuania; pipiniai@takas.lt, *Corresponding Author: antanas.kiveris@vpu.lt

Received 25 May 2010; revised 13 July 2010; accepted 20 July 2010.

\begin{abstract}
Phonon-assisted tunneling (PhAT) model is applied for explication of temperature-dependent conductivity and $I-V$ characteristics measured by various investigators for graphene nanoribbons and oxides ones. Proposed model describes well not only current dependence on temperature but also the temperature-dependent I-V data using the same set of parameters characterizing material under investigation. The values of active phonons energy and field strength for tunneling are estimated from the fit of current dependence on temperature and $I-V / T$ data with the phonon-assisted tunneling theory.
\end{abstract}

Keywords: Electronic Transport in Graphene; Phonon-Assisted Tunneling; Electron-Phonon Interaction

\section{INTRODUCTION}

Graphene systems, consisting of one or a few monolayer of carbon atoms connected via covalent bonds in the hexagonal lattice, have attracted great interest of researchers because of their peculiar physical and electrical properties and their potential for applications in nanoelectronics [1-3]. Graphene, in its basic two-dimensional (2D) form, does not have an energy gap separating the valence and conduction bands of graphene, which is an essential ingredient for making electronic devices. However, it is possible to induce the gap pattering single-layer graphene into nanometer size ribbons creating in this way one dimensional (1D) system similar to $1 \mathrm{D}$ carbon nanotubes $[4,5]$.

This energy gap depends on the width and crystallographic orientation of the graphene nanoribbon (GNR), and as was shown in [5] the induced gap's width varied from $300 \mathrm{meV}$ to $4 \mathrm{meV}$ with changing nanoribbons width from $15 \mathrm{~nm}$ to $90 \mathrm{~nm}$. Electronic conduction in GNR is a subject of intense study, both theoretical and experimental, during the past few years [5-19].

The charge carriers transport through GNR's shows some of intriguing peculiarities [19] and due to the band gap exhibits thermal activation behavior [5,13]. Thus, from this viewpoint, the conduction in GNR is similar to the conduction in conventional semiconductors. Thermally activated electrical conductivity is also observed in graphene oxide sheets [20-24], possessing also the energy band gap. The thermally activated conductivity in GNR and oxidized graphene by different authors is explained in a different way. For instance, Han et al. [13] thermal behavior of the conductivity in GNR at higher temperatures explained by thermal emission of the carriers from localized states in the band gap and by one dimensional variable range hopping (1D-VRH) at lower temperatures. Gómez-Navarro et al. [21] suggested that charge transport in individual chemically reduced graphene oxide sheet occurs via two-dimensional VRH between intact graphene islands. Kaiser et al. [22] the temperature-dependent intrinsic electrical conduction in individual monolayers of chemically reduced graphene oxide interpreted in the framework of the 2D-VRH in parallel with electric-field-driven tunneling at low temperatures. Graphene oxide thin film field effect transistors (GO-FET) fabricated by Jin et al. [23] on $\mathrm{Si}$ substrates showed $p$-type semiconducting behavior. The temperature dependence of the conductance of these films the authors [23] have explained by VRH with $2+3$ dimensionality, however, temperature-dependent $I-V$ characteristics were not explained. Thus, a variety of the interpretation of the temperature-dependent conductivity implies that the conduction mechanism is not fully understood.

We want to note that such behavior of the thermalactivated conductivity and temperature-dependent $I-V$ characteristics is usual for polymers and carbon nanotubes and in $[25,26]$ has been properly explained by the phonon-assisted tunneling (PhAT) model based on the quantum-mechanics [27]. Therefore, in this article we explore the PhAT model, which account phonon activation of the electric field stimulated tunneling emission of 
electrons from the local states to the conduction band, to describe the temperature-dependent conductance and $I-V$ characteristics observed in GNR structures and graphene oxides.

\section{THE PHONON-ASSISTED MODEL AND COMPARISON OF EXPERIMENTAL DATA}

We suggest that the source of the carriers is the electronic levels in the band gap of NRB at the metal-nanoribbon interface, the electrons from which enter into the conduction band due to the tunneling stimulated by phonons under action of the electric field. Assuming that due to the tunneling released electrons are transferred through the layer, the current will be equal to:

$$
I=e N W S,
$$

where $W$ is the phonon-assisted tunneling rate, $e$ is electronic charge unit, $N$ is the surface density of localized electrons, and $S$ is the area of the barrier electrode. On this basis we can compare the experimental data on current/conduction dependence upon applied voltage and temperature with computed tunneling rate dependence on field strength $E$ and temperature $W(E, T)$. For this purpose we will employ expression presented in $[25,26]$ which for the tunneling rate from the level of $\varepsilon_{T}$ depth gives:

$$
\begin{aligned}
& W=\frac{e E}{\left(8 m^{*} \varepsilon_{T}\right)^{1 / 2}}\left[\left(1+\gamma^{2}\right)^{1 / 2}-\gamma\right]^{1 / 2}\left[1+\gamma^{2}\right]^{-1 / 4} \times \\
& \exp \left\{-\frac{4}{3} \frac{\left(2 m^{*}\right)^{1 / 2}}{e E \hbar} \varepsilon_{T}^{3 / 2}\left[\left(1+\gamma^{2}\right)^{1 / 2}-\gamma\right]^{2} \times\right. \\
& \left.\left[1+\gamma^{1 / 2}+\frac{1}{2} \gamma\right]\right\}
\end{aligned}
$$

where $\gamma=\frac{\left(2 \mathrm{~m}^{*}\right)^{1 / 2} \Gamma^{2}}{8 e \hbar E \varepsilon_{T}^{1 / 2}}$ is a parameter, which provides the temperature dependence for tunneling process. Here $\Gamma^{2}=8 a(\hbar \omega)^{2}(2 n+1)$ is the width of the absorption band of the states broadened by the phonons, $n=\left[\exp \left(\hbar \omega / k_{B} T\right)-1\right]^{-1}$ is the temperature distribution of phonons, $\hbar \omega$ is the energy phonon taken part in the tunneling process, $m^{*}$ is the electron effective mass in the GNR lattice, and $a$ is the electron-phonon coupling constant, $\left(a=\Gamma_{o}^{2} / 8(\hbar \omega)^{2}\right)$.

At first in Figure 1 we present the fit of the experimental results on the temperature dependence of the conductivity in the temperature range from $4 \mathrm{~K}$ to $300 \mathrm{~K}$ measured by Han et al. [13] for GNR with the PhAT

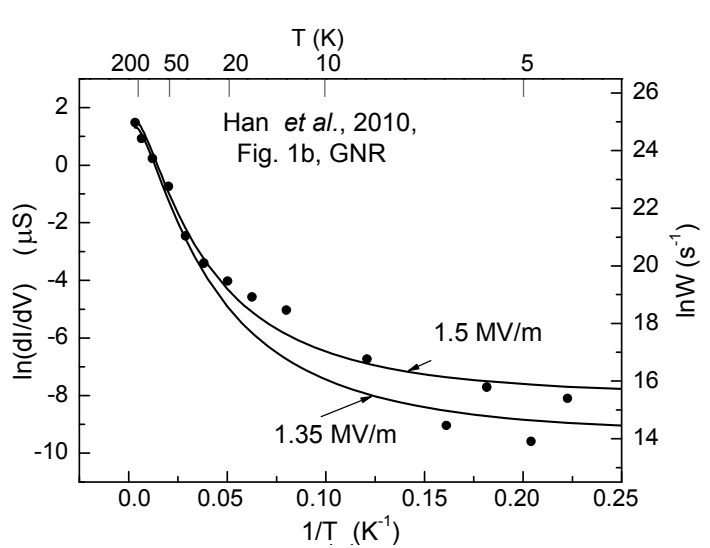

(a)

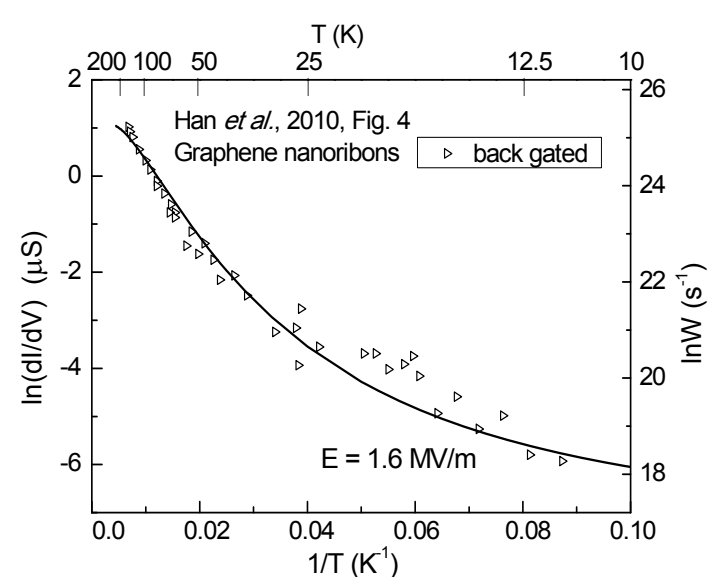

(b)

Figure 1. (a) The temperature dependence of the conductance minimum of GNR in the temperature range from 4 to $300 \mathrm{~K}$, extracted from [13, Figure 1(b)] (symbols) fitted to theoretical $\ln W(E, T)$ vs. $1 / T$ dependences (solid lines); (b) The same for back gated GNRs from Figure 4 in [13]. Data for computation: $\varepsilon_{T}=24 \mathrm{meV}, \hbar \omega_{1}=5 \mathrm{meV}, a_{1}=0.1, \hbar \omega_{2}=1 \mathrm{meV}, a_{2}=6$, $m^{*}=0.8 m_{e}$.

model. The authors [13] suggested thermally activated behavior at higher temperatures and 1D-VRH at lower temperatures. The computation of $W(E, T)$ for fitting with experimental data was performed using for $\varepsilon_{T}$ the value of $24 \mathrm{meV}$ assessed in [13], the effective mass of electron $m^{*}$ was taken to be equal to $0.8 \mathrm{~m}_{\mathrm{e}}$.

In carbon nanotubes there are exist a large variety of phonons [28]. We believe that this is and in GNRs samples. However, the energy of phonons taken part in the tunneling is unknown. Since the temperature dependence of the conductivity persists into low temperatures $(4 \mathrm{~K})$ and levels depth is small $(24 \mathrm{meV})$, the energy of phonons should be not be large. The phonons of higher energy, which probably dominate at higher temperatures, can be frozen in the low temperature range and therefore 
the phonons of low energy must be effective. For the calculation of $W(E, T)$ in this case we used phonons of 1 $\mathrm{meV}$ and $5 \mathrm{meV}$ energy, and the total $W(E, T)$ was expressed as a sum of $W_{1}(E, T)$ and $W_{2}(E, T)$ with $\hbar \omega_{1}=1$ $\mathrm{meV}$ and $\hbar \omega_{2}=5 \mathrm{meV}$, respectively. The electronphonon coupling constants $a_{1}$ and $a_{2}$ were chosen so that the best fit of the experimental data with the calculated dependences could be achieved. The fit of the $\ln (d I / d V)$ dependence on $1 / T$ extracted from [13, Figure 1(b) and Figure 4] with the theoretical dependence $\ln \left[W_{1}(E, T)+\right.$ $\left.W_{2}(E, T)\right]$ on $1 / T$ is shown by the solid line in Figures 1 (since the $W(E, T)$ is computed for one value of $E$ it was not divided by $E$ ). As is seen in Figure 1(a) and Figure 1(b), the theoretical curve describes well the temperature behavior of the conductivity in the entire range of the measured temperatures.

In Figure 2 the results on temperature dependence of the current measured by Kaiser et al. [22] for the monolayers of chemically reduced graphene oxide in the plot of $\ln I$ vs. $1 / T^{1 / 3}$ extracted from [22, Figures 3(a), (b), (c)] for three values of drain-source voltage $V_{\mathrm{ds}}$ and for two values of gate voltages $\mathrm{V}_{\mathrm{g}}=0$ and $\mathrm{V}_{\mathrm{g}}=-20 \mathrm{~V}$ are exposed. For the lowest value of bias voltage $\left(V_{\mathrm{ds}}=0.1 \mathrm{~V}\right)$, the measured data followed the 2D-VRH law (from 216 $\mathrm{K}$ down to $34 \mathrm{~K})$. For the largest bias voltage $\left(V_{\mathrm{ds}}=2 \mathrm{~V}\right)$, for all values of gate voltage there was a flattening below $\sim 100 \mathrm{~K}$, with temperature-independent behavior below $25 \mathrm{~K}$ down to the temperature of $2 \mathrm{~K}$. Such behavior of the experimental data the authors of [22] have described by the expression $G(T)=G_{1} \exp \left(-B / T^{1 / 3}\right)+G_{0}$, where the first term represents the usual 2D-VRH conduction expression and the second term represents purely tunneling conduction, i.e., independent of temperature. As can be seen in Figure 2, the theoretical curves computed using Eq.2 and phonon of $5 \mathrm{meV}$ energy only describe both the temperature-dependent and independent of temperature part of measured data equally well. This is because the phonons of $5 \mathrm{meV}$ are "frozen" at low temperatures and independent of temperature pure tunneling determines free carrier generation process. At higher temperatures the tunneling is by phonons stimulated process, consequently, temperature-dependent process.

Jin at al. [23] the temperature dependence of the conductance in graphene oxide thin films has explained by 2D- and 3D-VRH model. These experimental data in Figure 3(a) and Figure 3(b) are fitted to tunneling rate dependence on temperature also in natural logarithm of the $\ln \mathrm{W}(E, T)$ vs. $T^{-1 / 3}$ and $\ln \mathrm{W}(E, T)$ vs. $T^{1 / 4}$ plots.

A good fit with experimental data is achieved over all temperatures with parameters of $a=1.5, m^{*}=0.8 m_{e}$ and $\hbar \omega=12 \mathrm{meV}$, for $\varepsilon_{T}$ using the value of $0.1 \mathrm{eV}$ assessed in [23].

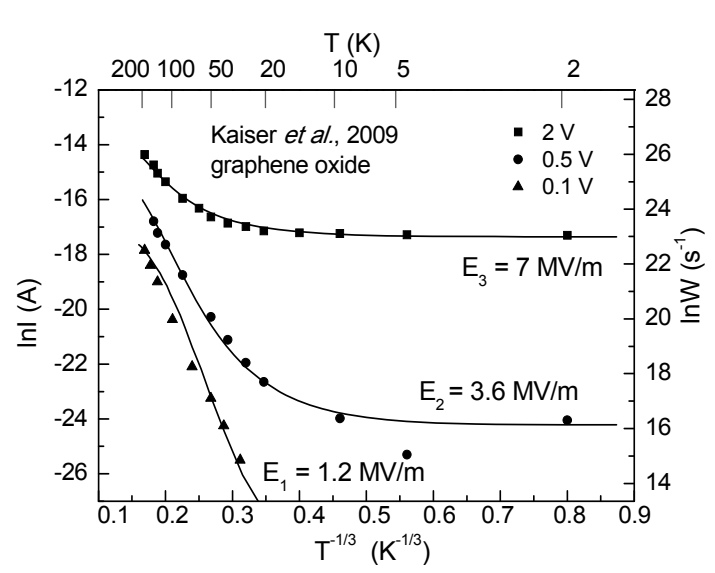

(a)

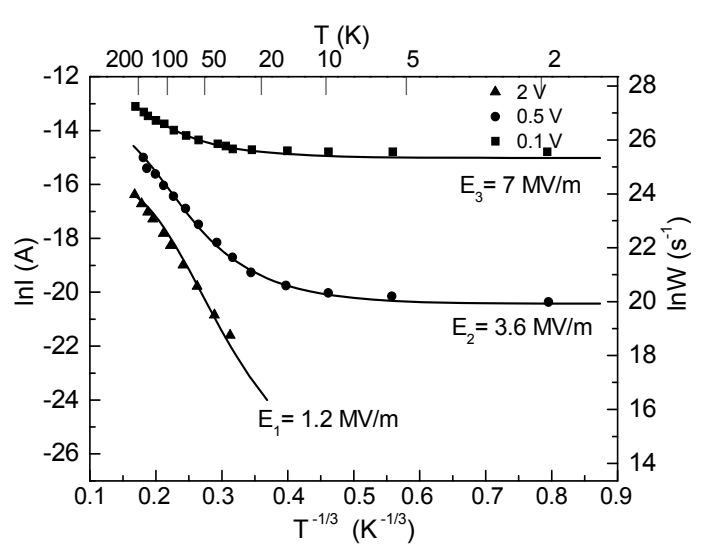

(b)

Figure 2. Natural logarithm of the source-drain current $I$ vs. $1 / T^{1 / 3}$ extracted from Figures 3(a), (b), (c) in [22], for different value of source-drain voltages $\mathrm{V}_{\mathrm{ds}}:$ (a) at the gate voltage $V_{g}=$ 0 (symbols), fitted to the theoretical PhAT $\ln W(E, T)$ dependences (solid lines); (b) The same at the gate voltage $\mathrm{Vg}=-20$ V. Parameters for PhAT computation: $\hbar \omega=5 \mathrm{meV}, a=1.5, m^{*}$ $=0.8 m_{e}$. and (a) $\varepsilon_{T}=40 \mathrm{meV}$, (b) $\varepsilon_{T}=33 \mathrm{meV}$. Note that in this case the $W(E, T)$ was computed using one value of the phonon energy.

Hence, all three models, i.e. 2D-VRH, 3D-VRH and $\mathrm{PhAT}$ in the temperature range from $280 \mathrm{~K}$ to $80 \mathrm{~K}$ explain the observed temperature dependence of the conductance well, but at lower temperatures, as can be seen in Figure 2 the VRH model mismatches. A deviation from the 2D-VRH model one can see at lower temperatures also in Figure 3. Thus, only from temperature dependence of the conductance cannot be resolved the conduction mechanism.

For decision on the dominant transport mechanisms more reliable are the $I-V$ characteristics and their variation with temperature. In particular such results from [23] 


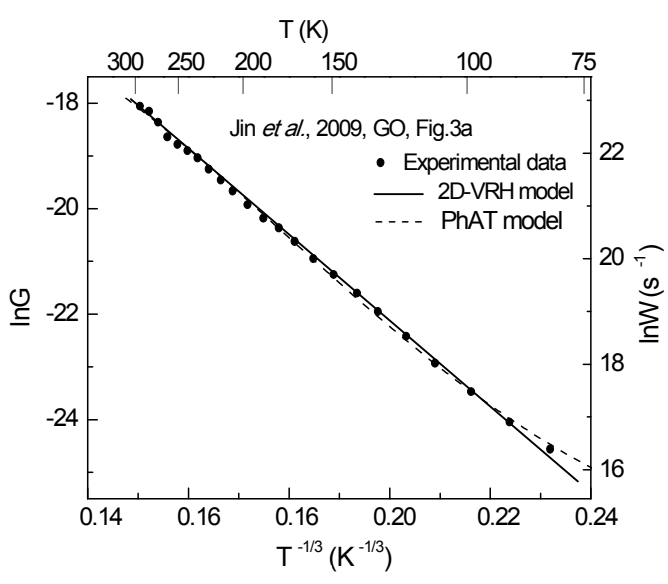

(a)

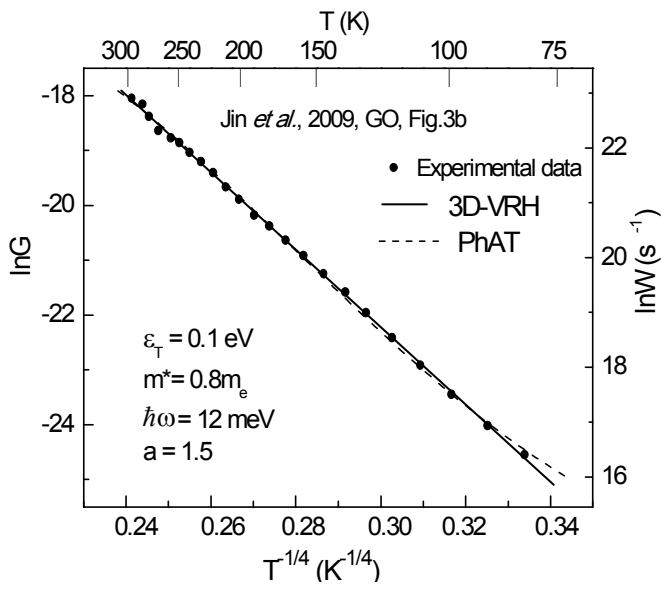

(b)

Figure 3. Natural logarithm of the measured conductance $G$ versus (a) $1 / T^{1 / 3}$ and (b) $T^{1 / 4}$ extracted from [23, Figure 3] (symbols), fitted to (a) 2D VRH, (b) 3D VRH model (solid line) and comparison with theoretical PhAT (a) $\ln W\left(E, T^{1 / 3}\right)$; (b) $\ln W\left(E, T^{1 / 4}\right)$ dependences (dashed lines). Parameters for computation: $\varepsilon_{T}=0.1 \mathrm{eV}, \hbar \omega=12 \mathrm{meV}, a=1.5, m^{*}=0.8 m_{e} . E=7$ $\mathrm{MV} / \mathrm{m}$.

we represent in the Figure 4. As can be seen in Figure 4, the theoretical dependences $W(E, T)$ computed using for three different temperatures and for the same parameters as in Figure 3 match very well with experimental data. We want to note that the difficulty arises in the framework of the VRH model explaining conductivity dependence on electric-field strength [29] therefore, for this dependence other models are used [29,30]. Thus, the results of the discussions can be consistently interpreted in the framework of the PhAT model.

\section{CONCLUSIONS}

In conclusion, the thermally activated conductivity in

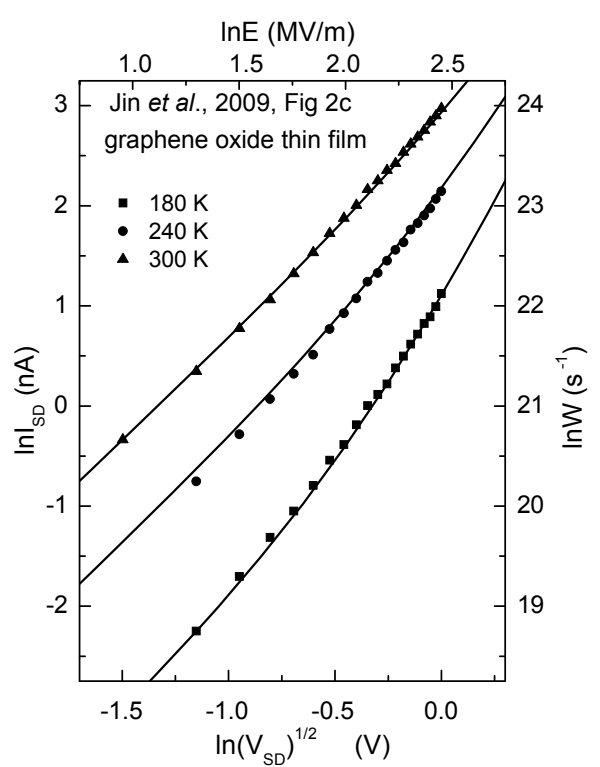

Figure 4. Natural logarithm of the measured $I_{S D}$ versus $\ln \left(V_{S D}\right)^{1 / 2}$ extracted from [23, Figure 3(c)] (symbols), fitted to theoretical PhAT $\ln W(E, T)$ dependences (lines) at three different temperatures. Parameters for computation: $\varepsilon_{T}=0.1 \mathrm{eV}, \hbar \omega=12 \mathrm{meV}, a=1.5, m^{*}=0.8 m_{e}$.

graphene nanoribbons and oxidized graphene can be explained by the temperature-dependent charge carrier generation thanks to phonon-assisted tunneling initiated by electrical field. In GNRs for conductance the phonons of low energy (1 meV) are also effective. Contrariwise, in graphene oxides the influence of phonons of low energy is not noticed. The phonon-assisted tunneling model describes also the temperature-dependent $I-V$ characteristics measured in oxidized graphene using the same set of parameters characterizing the material. From the fit of experimental data with the PhAT theory the field strength at which the tunneling occurs and participating in this process phonon's energy can be evaluated.

\section{REFERENCES}

[1] Novoselov, K.S., Geim, A.K., Morozov, S.V., Jiang, D., Zhang, Y., Dubonos, S.V., Grigorieva, I.V. and Firsov, A.A. (2004) Electric field effect in atomically thin carbon films. Science, 306(5696), 666-669.

[2] Service, R.F. (2009) Carbon sheets an atom thick give rise to graphene dreams. Science, 324(5929), 875-877.

[3] Castro Neto, A.H., Guinea, F., Peres, N.M.R., Novoselov, K.S. and Geim, A.K. (2009) The electronic properties of graphene. Reviews of Modern Physics, 81(1), 109-162.

[4] Berger, C., Song, Z., Li, X., Wu, X., Brown, N., Naud, C., Mayou, D., Li, T., Hass, J., Marchenkov, A.N., Conrad, E.H., First, P.N. and de Heer, W.A. (2006) Electronic confinement and coherence in patterned epitaxial graphene. Science, 312(5777), 1191-1196. 
[5] Han, M.Y., Özyilmaz, B., Zhang, Y. and Kim, P. (2007) Energy band-gap engineering of graphene nanoribbons. Physical Review Letters, 98(20), 206805.

[6] Yan, Q., Huang, B., Yu, J., Zheng, F., Zang, J., Wu, J., Gu, B.-L., Liu, F. and Duan, W. (2007) Intrinsic currentvoltage characteristics of graphene nanoribbon transistors and effect of edge doping. Nano Letters, 7(6), 1469 1473.

[7] Murali, R., Yang, Y., Brenner, K., Beck, T. and Meindl, J.D. (2009) Breakdown current density of graphene nanoribbons. Applied Physics Letters, 94(24), 243114.

[8] Li, Z., Qian, H., Wu, J., Gu, B.-L. and Duan, W. (2008) Role of symmetry in the transport properties of graphene nanoribbons under bias. Physical Review Letters, 100 (20), 206802.

[9] Rosales, L., Orellana, P., Barticevic, Z. and Pacheco, M. (2008) Transport properties of graphene nanoribbon heterostructures. Microelectronics Journal, 39(3-4), 537-540.

[10] Rigo, V.A., Martins, T.B., da Silva, A.J.R., Fazzio, A. and Miwa, R.H. (2009) Electronic, structural, and transport properties of Ni-doped graphene nanoribbons. Physical Review B, 79(7), 075435.

[11] Sinitskii, A., Fursina, A.A., Kosynkin, D.V., Higginbotham, A.L., Natelson, D. and Tour, J.M. (2009) Electronic transport in monolayer graphene nanoribbons produced by chemical unzipping of carbon nanotubes. Applied Physics Letters, 95(25), 253108.

[12] Xie, Y.E., Chen, Y.P. and Zhong, J.X. (2009) Electron transport of folded graphene nanoribbons. Journal of Applied Physics, 106(10), 103714.

[13] Han, M.Y., Brant, J.C. and Kim. P. (2010) Electron transport in disordered graphene nanoribbons. Physical Review Letters, 104(5), 056801.

[14] Zhang, Y.-Y., Hu, J.-P., Xie, X.C., Liu, W.M. (2009) Abnormal electronic transport in disordered graphene nanoribbon. Physica B: Condensed Matter, 404(16), 22592262.

[15] Molitor, F., Stampfer, C., Güttinger, J., Jacobsen, A., Ihn, T. and Ensslin, K. (2010) Energy and transport gaps in etched graphene nanoribbons. Semiconductor Science and Technology, 25(3), 034002.

[16] Ihnatsenka, S. and Kirczenow, G. (2009) Conductance quantization in strongly disordered graphene ribbons. Physical Review B, 80(20), 201407(R).

[17] Lian, Ch., Tahy, K., Fang, T., Li, G., Xing, H.G. and Jena, D. (2010) Quantum transport in graphene nanoribbons patterned. Applied Physics Letters, 96(10), 103109.

[18] Jiménez, D. (2008) A current-voltage model for Schottky-barrier graphene-based transistors. Nanotechnology,
19(34), 345204.

[19] Wakabayashi, K., Takane, Y., Yamamoto, M. and Sigrist, M. (2009) Electronic transport properties of graphene nanoribbons. New Journal of Physics, 11(9), 095016.

[20] Jung, I., Dikin, D.A., Piner, R.D. and Ruoff, R.S. (2008) Tunable electrical conductivity of individual graphene oxide sheets reduced at "Low" temperatures. Nano Letters, 8(12), 4283-4287.

[21] Gómez-Navarro, C., Weitz, R.T., Bittner, A.M., Scolari, M., Mews, A., Burghard, M. and Kern, K. (2007) Electronic transport properties of individual chemically reduced graphene oxide sheets. Nano Letters, 7(11), 34993503.

[22] Kaiser, A.B., Gómez-Navarro, C., Sundaram, R.S., Burghard, M. and Kern, K. (2009) Electrical conduction mechanism in chemically derived graphene monolayers. Nano Letters, 9(5), 1787-1792.

[23] Jin, M., Jeong, H.-K., Yu, W.J., Bae, D.J., Kang, B.R. and Lee, Y.H. (2009) Graphene oxide thin film field effect transistors without reduction. Journal of Physics D: Applied Physics, 42(13), 135109.

[24] Shao, Q., Liu, G., Teweldebrhan, D. and Balandin, A.A. (2008) High-temperature quenching of electrical resistance in graphene interconnects. Applied Physics Letters, 92(20), 202108.

[25] Pipinys, P., Rimeika, A. and Lapeika, V. (2004) DC conduction in polymers under high electric field. Journal of Physics D: Applied Physics, 37(6), 828-831.

[26] Pipinys, P. and Kiveris, A. (2008) Phonon-assisted tunnelling in electrical conductivity of individual carbon nanotubes and networks ones. Physica B: Condensed Matter, 403(19-20), 3730-3733.

[27] Kiveris, A., Kudžmauskas Š. and Pipinys P. (1976) Release of electrons from traps by an electric field with phonon participation. Physica Status Solidi A, 37(1), 321327.

[28] Popov, V.N., Henrard, L. and Lambin, P. (2005) Electron-phonon and electron-photon interactions and resonant Raman scattering from the radial-breathing mode of single-walled carbon nanotubes. Physical Review B, 72(3), 035436.

[29] Wang, D.P., Feldman, D.E., Perkins, B.R., Yin, A.J., Wang, G.H., Xu, J.M. and Zaslavsky, A. (2007) Hopping conduction in disordered carbon nanotubes. Solid State Communication, 142(5), 287-291.

[30] Kaiser, A.B. and Park, Y.W. (2005) Current-voltage characteristics of conducting polymers and carbon nanotubes. Synthetic Metals, 152(1-3), 181-184. 